\title{
ESTUDO DO COMPORTAMENTO DE MATERIAIS POROSOS EM CONCRETOS DE ALTA RESISTÊNCIA DIANTE DE ELEVADAS TEMPERATURAS
}

\author{
CAETANO, LUCIANE FONSECA \\ Engenheira Civil, Dra. \\ Universidade Federal do Rio Grande do Sul \\ Rio Grande do Sul; Brasil \\ lucianefc@gmail.com

\section{PERONDI, MATHIAS} \\ Engenheiro Civil \\ Universidade Federal do Rio Grande do Sul \\ Rio Grande do Sul; Brasil \\ mathiasperondi@gmail.com
}

\author{
RISBACIK, FELIPE \\ Graduando em Engenharia Civil \\ Universidade Federal do Rio Grande do Sul \\ Rio Grande do Sul; Brasil \\ feliperisbacik@hotmail.com \\ SILVA, SURIANE DE SOUZA DA \\ Graduanda Engenharia Civil \\ Universidade Federal do Rio Grande do Sul \\ Rio Grande do Sul; Brasil \\ surianesilva@gmail.com
}

\author{
SILVA FILHO, LUIZ CARLOS PINTO DA \\ Professor PhD em Engenharia Civil \\ Universidade Federal do Rio Grande do Sul \\ Rio Grande do Sul; Brasil \\ 1carlos66@gmail.com
}

\section{RESUMO}

Analisando a literatura recente é possível verificar um aumento exponencial do número de pesquisas relacionados ao concretos de alto resistência (CAR), que se caracterizam por uma relação água/cimento reduzida, maior consumo de cimento e uma matriz mais compacta. Porém, este tipo de mistura de alta compacidade e baixa relação água/cimento tende a apresentar uma maior probabilidade de fissuração nas primeiras idades, devido à maior suscetibilidade à retração autógena. Uma alternativa de controle com resultados interessantes é o uso de cura interna, como evidenciado em Caetano (2019). Esta técnica busca utilizar materiais porosos com características adequadas para atuar como reservatórios de água internos na matriz cimentícea. Sob o aspecto do controle de fissuração a cura interna é claramente positiva. Considerando, entretanto, que os agregados ocupam cerca de 70 a $80 \%$ do volume total de concreto e, portanto, influenciam fortemente seu comportamento térmico, pouco se conhece sobre o comportamento de misturas cimentícias compactas, compostas com agregados porosos saturados expostas a elevadas temperaturas. Esse artigo descreve resultados de uma pesquisa em desenvolvimento visando avaliar o comportamento de misturas de CAR com agregados porosos usados por Caetano (2019) com sucesso para cura interna em altas temperaturas. Foram avaliadas cinco misturas, geradas a partir de um traço de referência com proporções 1:1,914 de cimento:agregado miúdo, com adição de $10 \%$ de sílica ativa e relação a/c de 0,30 . Tres outras misturas foram elaboradas com substituição parcial do agregado miúdo por agregados porosos tipo cinza pesada, casca cerâmica e coríndon parcialmente saturados. Um traço de controle com a água total incorporada no agregado foi usada como referência secundária $(\mathrm{a} / \mathrm{c}=$ 0,36). Para a análise térmica foram usados os patamares de temperatura de $23^{\circ} \mathrm{C}$ (ambiente), $400^{\circ} \mathrm{C}$ e $800^{\circ} \mathrm{C}$, com duas taxas de aquecimento: $2^{\circ} \mathrm{C} / \mathrm{min}$ (lenta) e $27,5^{\circ} \mathrm{C} / \mathrm{min}$ (rápida).

Palavras Chave: Cura Interna; Concreto de alta resistência; Altas temperaturas; Agregados porosos.

\begin{abstract}
Looking into recent literature it is possible to verify an exponential increase in the number of scientific research associated with High Strength Concrete (HSC), mixes that are characterized by a low water/cement ratio, higher cement consumption and a compact matrix. However, this kind of low porosity concrete tends to present a higher cracking probability at early age, because of the increased susceptibility to autogenous shrinkage. The use of internal curing (IC) is an attractive solution to this problem, which has shown interesting potential, as demonstrated in Caetano (2019). This IC technique seeks to use a small portion of porous aggregate with adequate properties to act as an internal water reservoir in highly densified cement matrices. To control early cracking IC has shown great promise but, considering that aggregates occupy around 70 to $80 \%$ of concrete volume and have great influence on thermal behavior, there is yet very little knowledge about the behavior of compact cement mixtures made of saturated porous aggregate at high temperatures. This paper describes data from a study focused on investigating experimentally the behavior of HSC mixes with porous aggregate used by Caetano (2019) exposed to high temperatures. Five samples were evaluated, adapted from a reference HSC made using a 1:1,914 (cement: fine aggregate) proportion, with the addition of 10\% of active silica fume and a w/c ratio of 0,30. A control mix was made with w/c ratio 0,36, representing the total amount of water included in the porous aggregate. Three other mix were made with the partial replacement of fine aggregate with porous materials (coal burning ash, ceramic moulding tailing e corindon). Tests were conducted at room temperature $\left(23^{\circ} \mathrm{C}\right), 400^{\circ} \mathrm{C} ; 800^{\circ} \mathrm{C}$, using two different heating rates: $2^{\circ} \mathrm{C} / \mathrm{min}$ (slow) and $27,5^{\circ} \mathrm{C} / \mathrm{min}$ (rapid).
\end{abstract}

Keywords: Internal Curing; High Performance Concrete; High Temperature; Lightweight aggregate. 


\section{INTRODUÇÃO}

Hoje em dia, percebe-se um interesse crescente no uso de misturas cimentícias de reduzida relação a/c. Em geral os resultados para este tipo de mistura são excelentes em termos de resistência à compressão e quanto à durabilidade. Todavia, percebeu-se que este tipo de concreto apresentava maior suscetibilidade à fissuração nas idades iniciais. Após estudos se concluiu que o aparecimento de fissuras nas primeiras idades, em concretos com baixa relação água cimento, está frequentemente relacionado ao fenômeno de retração autógena.

A retração autógena pode ser considerada como um dos principais calcanhares de aquiles dos concretos de alta resistência, junto com o eventual desplacamento explosivo de origem térmica. O aparecimento de fissuras de retração em baixas idades pode reduzir a vida útil das estruturas de concreto, pois a presença das fissuras facilita a entrada de agentes agressivos por um atalho, podendo prejudicar a elevada durabilidade das matrizes de alta compacidade.

Segundo Kovler e Jensen (2007) a retração autógena é normalmente desprezível para concretos com relações a/c (água/cimento) maiores que 0,40. Entretanto, com a gradativa redução da relação a/c, a retração autógena tende a aumentar rapidamente, tornando-se um fator importante na retração total. De acordo com a literatura, a retração autógena em concretos com a/c de 0,30 pode chegar a representar metade da retração total.

Uma das formas de reduzir os efeitos da retração, em geral, consiste na aplicação de procedimentos de cura. Porém as técnicas de cura externa, que são amplamente utilizadas e exitosas, para concretos de relação a/c mais elevada, se mostram ineficazes para misturas com baixa relação a/c $(<0.40)$, dada a grande compacidade de sua microestrutura, caracterizada por uma estrutura de poros refinada e muitas vezes desconectada. Como explica Bentur (2000), a cura externa atinge apenas poucos milímetros superficiais da estrutura, cerca de $50 \mathrm{~mm}$, em concretos mais densos.

Nas últimas duas décadas um conjunto de pesquisas se deteve em uma alternativa relativamente inovadora, denominada cura interna. A noção fundamental é que para reduzir ou mitigar a retração autógena seria interessante gerar reservatórios internos de água que pudessem suprir a demanda hídrica ao longo da hidratação sem acarretar em perdas de resistência ou durabilidade. A técnica se caracteriza pelo uso de um agente de cura interna que serve como reservatório de água, liberando gradualmente a mesma durante a hidratação, conforme as condições específicas demandem. Normalmente se usam para esse fim alguns materiais porosos, em substituição a uma pequena parcela dos agregados ou o uso de SAP (polímeros superabsorventes) entudados por Assman (2013), Manzano (2016), entre outros.

Neste trabalho avaliou-se o efeito do uso de agregado poroso saturado em relação ao comportamento frente a elevadas temperaturas. Segundo Hager (2013) os agregados ocupam de 70 a 80\% do volume de concreto e, portanto, influenciam fortemente seu comportamento térmico.

Conforme relatado por Almeida (2017) a porosidade total do concreto aumenta com o aumento da temperatura. A redução do volume das fases hidratadas devido à perda de água leva à formação de vazios que contêm ar e ao aumento da estrutura de poros, reduzindo a rigidez e a resistência do concreto. Entre $100^{\circ} \mathrm{C}$ e $300^{\circ} \mathrm{C}$, a porosidade aumenta devido à desidratação do gel de C-S-H. Para temperaturas mais elevadas, a dimensão dos poros aumenta devido ao aparecimento de microfissuras causadas pela incompatibilidade térmica entre agregado e matriz (SANTOS, 2012).

De acordo com Paulon (1984), quando submetido à temperaturas de até $150^{\circ} \mathrm{C}$, o concreto não sofre alteração sensível de sua resistência, podendo chegar a níveis de decréscimo em torno de $70 \%$, aos $600^{\circ} \mathrm{C}$, devido à desidratação do gel e ao aumento da microfissuração. A resistência à compressão não sofre alteração apreciável até cerca de $300^{\circ} \mathrm{C}$. Entretanto, a partir deste valor, uma redução considerável se inicia, assumindo uma perda em torno de $20 \%$. Ensaios realizados por Galleto \& Meneguini (2000), apresentaram, para o concreto convencional aquecido a $300^{\circ} \mathrm{C}$ e resfriado lentamente, uma perda de resistência à compressão de $24 \%$ em relação à sua resistência original (sem aquecimento).

Para Khoury et al. (2002) os principais fatores que afetam a resistência residual do concreto em altas temperaturas são a composição do concreto, o teor de umidade, as condições de ensaio, a taxa de aquecimento, a taxa de resfriamento, o tempo de exposição a altas temperaturas. Contudo, há um consenso na literatura, que independente das condições de ensaio, de que a resistência diminui com o aumento da temperatura e essa redução é mais acentuada para temperaturas acima de $400^{\circ} \mathrm{C}$.

Além da deterioração das fases e consequentemente alteração do comportamento da mistura há a possibilidade de ocorrência de spalling. De acordo com Malhotra (1982), o spalling é provocado pelo desenvolvimento de altas pressões 
de vapor nos poros, com formação de fissuras no interior do concreto, paralelas à superfície. Logo, sob determinadas condições de tensão, ocorre o arrancamento explosivo das camadas superficiais do concreto. Para alguns autores como Hentz (1992) e Costa et al (2002), o lascamento explosivo ocorre habitualmente para taxas de aquecimento mais elevadas, superiores a $10^{\circ} \mathrm{C} / \mathrm{min}$, podendo ser inferior para misturas com adições pozolânicas.

Dito isto, o presente trabalho tem por objetivo principal avaliar a influência do uso de agregados porosos saturados, utilizados como agentes de cura interna, sob ação de altas temperaturas, em misturas cimentícias de alta resistência. Busca-se verificar o comportamento tanto da resistência residual dos microconcretos aquecidos até $800^{\circ} \mathrm{C}$, quanto em relação à ocorrência de spalling.

\section{PROGRAMA EXPERIMENTAL}

Neste item serão apresentadas a descrição e caracterização do material ensaiado, bem como a metodologia adotada para a obtenção de dados relevantes para o atendimento dos objetivos propostos.

\subsection{Material}

Por se tratar de uma pesquisa que utiliza agentes de cura interna para redução da retração autógena, o traço utilizado foi baseado nas especificações do RILEM TC 225-SAP-Newsletter $n^{\circ} 1$, que descreve uma proporção padrão de mistura usada para permitir que alguns trabalhos que investigaram a ação de polímeros superabsorsores (no inglês, super absorbant polymers - SAP) como agentes de cura interna pudessem ser comparados entre si. Apesar desta pesquisa não se utilizar SAP, foi decidido que seria interessante usar a mesma proporção de mistura, para que fosse possível realizar comparações entre os resultados deste trabalho e outras pesquisas de cura interna usando outras técnicas.

Destaca-se que forma necessárias pequenas alterações no traço padrão, de forma a adequar o mesmo aos materiais locais, buscando manter as características próximas às do traço original recomendado. Tomando como base o traço recomendado, foi utilizado para a mistura de referência uma proporção 1:1,914 de cimento e areia, sendo usada uma adição de $10 \%$ de sílica ativa, em relação a massa de cimento, e uma relação a/c de 0,30. O teor de aditivo plastificante foi definido de forma que o espalhamento na mesa de graff atingisse $350 \pm 20 \mathrm{~mm}$, quando ensaiado conforme Norma NBR 13276 (2016) e $250 \pm 20 \mathrm{~mm}$, quando ensaiado sem quedas nem forças aplicadas. Esta metodologia foi a mesma utilizada por Assmann (2013) e Sensale e Gonçalves (2014) em seus trabalhos.

Para a confecção dos microconcretos (essa denominação é utilizada por tratar-se de uma mistura sem agregado graúdo, porém com características mecânicas de concreto) foram utilizados cimento Portland de Alta Resistência Inicial, classificado como CPV-ARI, da marca Nacional; areia regular extraída do Rio Jacuí, diâmetro máximo 4,8 mm e módulo de finura 2,4; sílica ativa em pó da marca Ferbrasa e aditivo superplastificante TecFlow 8000.

Foram avaliados três materiais distintos para utilização como agregados porosos: casca cerâmica, cinza pesada e corindon - os dois primeiros são resíduos industriais. Os materiais podem ser observados na Figura 1.

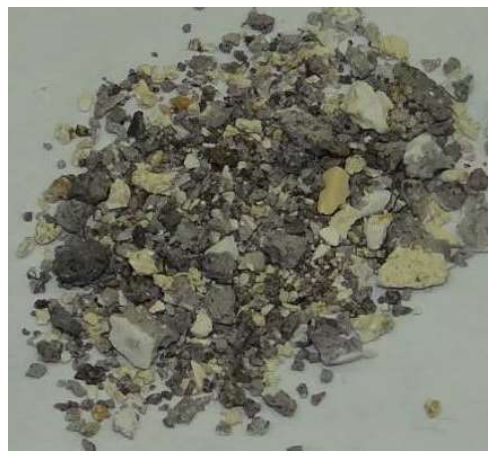

(a)

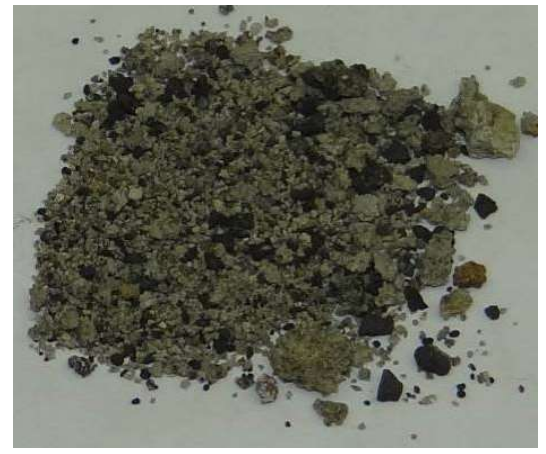

(b)

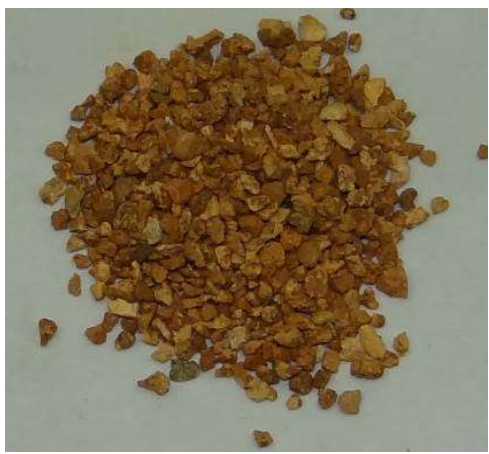

(c)

Figura 1: Aspecto do agregado poroso: (a) casca cerâmica; (b) cinza pesada e (c) corindon.

Cabe aqui gerar uma breve resenha sobre estes materiais porosos. A casca cerâmica é obtida pelo pós-processamento por cominuição de um resíduo da indústria siderúrgica, resultante do descarte do material cerâmico usado como molde para microfusão de peças metálicas; a cinza pesada é um resíduo do processo de queima do carvão, sendo essa a fração mais 
grossa e granular que encontra-se decantada no fundo do queimador e; o corindon artificial ativado, tipo de bauxita calcinada que possui cerca de $89 \%$ de $\mathrm{Al}_{2} \mathrm{O}_{3}$ em sua composição, é comercializado para fins de clarificação e regeneração de óleos, sendo empregado também para contenção de vazamentos de óleo.

A quantidade de água a ser usada para fins de cura interna, ou seja, a quantidade de água de saturação do agregado porção de água que busaca-se "reservar" no agregado para ser liberada para garantir a hidratação completa do concreto foi estimada com base no modelo de Powers. De acordo com o modelo de Powers a quantidade de água necessária para cura interna depende da relação a/c inicial e da quantidade de sílica ativa. Para uma relação a/c de 0,3 e uma adição de $10 \%$ de sílica ativa, a quantidade de água estimada pelo modelo, seria de 0,066 kg para cada $1 \mathrm{~kg}$ de cimento, isto é, um valor fixo.

Esta quantidade fixa, adicional, de água é inserida no agregado poroso, que substitui parcialmente a areia em função da capacidade de absorção de água de cada material. Para o cálculo da substituíção foram necessárias as determinações de massa específica aparente e da taxa de absorção, em 24 h de imersão em água. Tanto a massa específica aparente, quando a determinação da taxa de absorção foram executadas seguindo as recomendações das Normas NM 52(2003) e ASTM C1761 (2015), porém com adaptação, pois a determinação do ponto de saturação superfície seca foi efetuada com o uso de papel toalha, maiores detalhes podem ser obtidos no trabalho de Caetano (2019). Na Tabela 1 é possível verificar os valores de massa aparente, taxa de absorção e o porcentual de substituição volumétrica.

Tabela 1: Massa específica aparente, taxa de absorção e valores de substituição do agregado poroso pela areia.

\begin{tabular}{c|c|c|c}
\hline Agregado & $\begin{array}{c}\text { Massa específica } \\
\text { aparente }\left[\mathrm{kg} / \mathrm{dm}^{3}\right]\end{array}$ & $\begin{array}{c}\text { Taxa de absorção } \\
{[\%]}\end{array}$ & $\begin{array}{c}\text { Substituição } \\
\text { volumétrica [\%] }\end{array}$ \\
\hline Areia & 2,532 & 4,4 & - \\
\hline Casca Cerâmica & 2,330 & 8,5 & 45 \\
\hline Cinza Pesada & 1,377 & 18,9 & 34 \\
\hline Corindon & 1,667 & 31,2 & 17 \\
\hline
\end{tabular}

Como pode ser visto na Tabela 1, quanto menor a taxa de absorção de água, em 24 h de imersão, maior o percentual de substituição da areia pelo material poroso, visto a necessidade de assegurar que o volume de agregado poroso fosse capaz de armazenar $0,066 \mathrm{~kg}$ de água para cada $1 \mathrm{~kg}$ de cimento utilizado.

No total foram avaliadas cinco misturas distintas, sendo duas delas de referência, ambas com traço padrão recomendado pela RILEM, porém uma com relação a/c de 0,30 e outra com a/c de 0,366 (relação a/c total utilizada nas misturas com agregados porosos). As demais misturas foram executadas com substituíção parcial da areia pelo material poroso saturado (com saturação de $\mathrm{a} / \mathrm{c}_{\text {int }} 0,066$ )

\subsection{Metodologia}

A seguir serão apresentadas as metodologias adotadas para moldagem dos corpos de prova, bem como para a realização da etapa de aquecimento e de avaliação mecânica dos CPs.

\subsubsection{Moldagem e Estabilização da Umidade dos Corpos de Prova}

Anteriormente a execução da mistura e moldagem dos corpos de provas, os agregados porosos foram imersos em água por $24 \mathrm{~h}$. A quantidade de água colocada no recipiente de imersão do material poroso foi o total de água que seria utilizada na mistura (ou seja, a parcela de água calculada pela relação água-cimento mais 100\% da água de cura interna determinada a partir do modelo de Powers). Apesar de alguns pesquisadores (Lura, 2003; Sensale e Gonçalves, 2014) relatarem que a simples imersão em água não é efetiva para obtenção da plena saturação dos agregados porosos, esta opção foi adotada por questões logísticas, no caso de uso prático.

As misturas dos materiais, para fabricação dos microconcretos, foram executadas em uma argamassadeira vertical, com capacidade útil de 20 litros. Após procedimento da mistura, o microconcreto foi avaliado quanto ao espalhamento e posteriormente utilizado para moldagem dos corpos de prova. 
Os corpos de prova foram moldados em formas metálicas prismáticas de $75 \times 75 \times 285 \mathrm{~mm}$, como pode ser observado na Figura 2. Logo após a moldagem as fôrmas foram envoltas em filme de PVC para dificultar a perda de água por evaporação. Vinte quatro horas após a moldagem os mesmos foram desmoldados e curados por 27 dias em água com cal. Posterior ao processo de cura, os CPs foram dispostos em uma prateleira, de forma que todas as faces dos cps ficassem em contato com ar, em uma sala climatizada, com temperatura de $23^{\circ} \mathrm{C} \pm 2{ }^{\circ} \mathrm{C}$ e uma UR de $50 \% \pm 5 \%$, para que ocorresse o equilíbrio de umidade. Os corpos de prova foram mandidos em ambiente climatizado por, pelo menos 2 anos. Para a exposição dos cps ao aquecimento, os mesmos foram fragmentados em elementos cúbidos de $75 \mathrm{~mm}$ de aresta. Destacase que a fragmentação foi efetuada com corte à seco, de forma a alterar o equilíbrio de umidade dos mesmos.

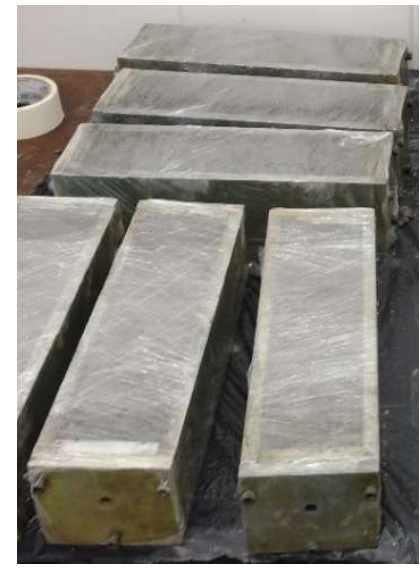

Figura 2: Corpos de prova moldados e embalados com filme de PVC.

\subsubsection{Aquecimento}

Para a avaliação do comportamento do microconcreto frente à ocorrência de spalling e em termos de resistência residual após aquecimento, os cps foram submetidos a patamares de temperatura de $23^{\circ} \mathrm{C}$ (ambiente), $400^{\circ} \mathrm{C}$ e $800^{\circ} \mathrm{C}$. Segundo diversas pesquisas, tais como Almeida (2017), Lee (2016) e Peng (2005), a resistência do concreto após aquecimento pode sofrer incrementos para temperaturas de até $200^{\circ} \mathrm{C}$, tendendo a apresentar de forma consistente reduções a partir de $400^{\circ} \mathrm{C}$, quando se inicia a degradação dos compostos que forma a estrutura de poros e a desidratação do hidróxido de cálcio. Dado esse limiar, decidiu-se iniciar o estudo com um patamar de exposição de $400^{\circ} \mathrm{C}$, no qual já se esperava obter reduções de resistência bem claras para o traço referência. O segundo patamar de $800^{\circ} \mathrm{C}$ foi escolhido por que, em muitos estudos, a faixa de $800^{\circ} \mathrm{c}$ a $900^{\circ} \mathrm{c}$ é usada como temperatura máxima, pois nesse patamar a resistência residual já está muito reduzida, mas os corpos de prova ainda tendem a estar íntegros, possibilitando a realização de ensaios que permitem uma melhor comparação entre os resultados.

De forma a melhor avaliar os efeitos de interesse desse trabalho, foram adotadas duas taxas de aquecimento, uma mais lenta, que permite um avanço gradual da temperatura para o interior dos corpos de prova, favorecendo a homogeneização de condições e a avaliação da resistência residual dos cps, e outra, mais rápida, que favorece a geração de diferenciais de temperatura e a ocorrência de desplacamentos explosivo (explosive spalling). De acordo com Kichhoff (2010), a ocorrência de desplacamento explosivo se dá para condições de exposição específicas, quando a umidade interna é superior a 70\% e temos um aquecimento rápido, que gera diferenciais significativos na temperatura e na formação de vapor na estrutura de poros. Esse fenômeno é decorrente da acumulação de poropressão e se desenvolve até cerca de 30 min do início do aquecimento, antes da fissuração intensa da matriz (que permite o alívio da poropressão), como descreve Khoury et al. (2002).

O desplacamento explosivo é um dos fenômenos preocupantes em estruturas de alta resistência e necessita ser evitado ou mitigado, para que não ocorram danos significativos nas mesmas em caso de incêndio. Um dos objetivos do estudo era justamente avaliar se a presença da parcela de agregados porosos (e a eventual densificação da área em torno dos mesmos devido à cura interna) poderia impactar de alguma forma no comportamento de explosive spalling. Por isso optou-se por trabalhar com duas taxas de aquecimento, sendo uma delas similar à prevista na ISO 834:2014 - "Fire resistance tests", da ordem de $27,5^{\circ} \mathrm{C} / \mathrm{min}$. Com a reduzida relação a/c, uso de sílica e aquecimento rápido se desejava induzir o desplacamento explosivo no traço de referência, e verificar se a cura interna com uso de agregados porosos poderia agravar ou mitigar o fenômeno nos demais traços. 
Já para melhor avaliar a resistência residual e o comportamento em incêndios mais realistas se decidiu usar também uma taxa de aquecimento lenta, da ordem de $2{ }^{\circ} \mathrm{C} / \mathrm{min}$, conforme recomendação do RILEM TC 129-MHT: "Test methods for mechanical properties of concrete at high temperatures". Esta taxa foi definida em função do tamanho do corpo de prova analisado.

O uso de taxa de aquecimento mais lenta favorece a homogeneidade de temperatura em toda a massa do corpo de prova, isto é, que a temperatura seja similar na superfície e na parte central do cp.

A Figura 3 apresenta um esquema ilustrativo indicando as temperaturas e taxas de aquecimento utilizadas neste trabalho.

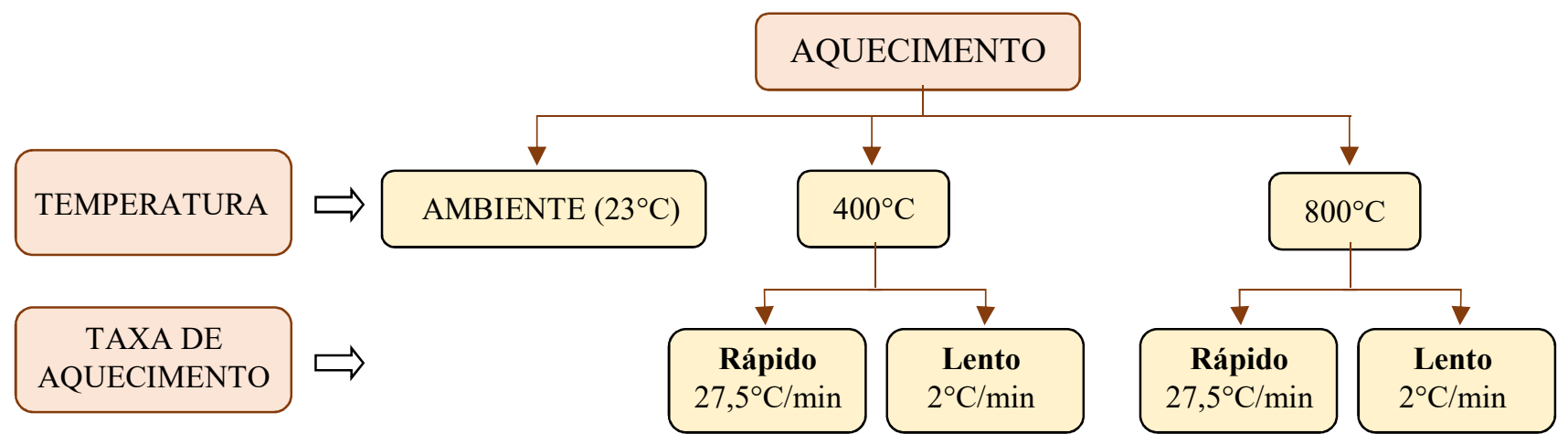

Figura 3: Fluxograma da metodologia de aquecimento adotada neste trabalho.

Para definir quanto tempo seria necessário para equalizar a temperatura de exposição com a temperatura do núcleo do corpo de prova, anteriormente aos testes principais do programa experimental, dois CPs pilotos contendo termopares tipo $\mathrm{K}$ foram aquecidos, respectivamente a $400^{\circ} \mathrm{C}$ e $800^{\circ} \mathrm{C}$, para determinação do tempo necessário de exposição até o equilíbrio entre as temperaturas interna e externa. Foram usados dois termopares para avaliar esse momento, um posicionado na parte central do exemplar e o outro na face do mesmo, conforme pode ser observado na Figura 4.

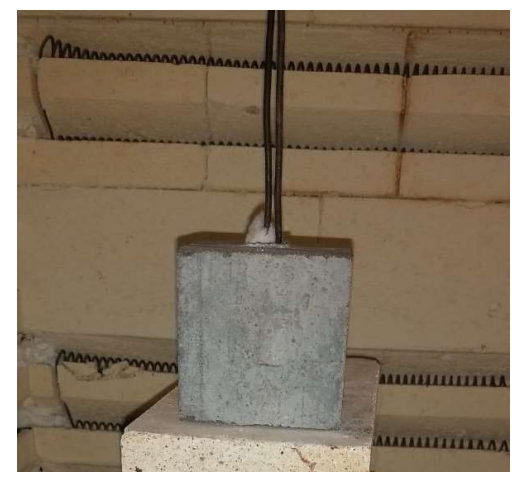

Figura 4: Fluxograma da metodologia de aquecimento adotada neste trabalho.

Após a análise dos resultados preliminares verificou-se, para a taxa de aquecimento de $2^{\circ} \mathrm{C} / \mathrm{min}$, que o tempo para que as temperaturas entrassem em equilíbrio era de $45 \mathrm{~min}$, sendo este o tempo definido de permanência na temperatura especificada. Já para a taxa de aquecimento de $27,5^{\circ} \mathrm{C} / \mathrm{min}$, o tempo necessário para equilíbrio foi de 30 min, determinado conforme procedimento utilizado por Lima (2005).

Destaca-se que durante os ensaios os corpos de prova foram posicionados no forno elétrico, de forma que cada um ficasse com todas as faces expostas ao calor, com circulação de ar entre eles. Dadas as dimensões dos fornos elétricos empregados, isso permitiu alocar 9 CPs por ensaio, três de cada mistura. Para que fosse avaliada a possível influência do posicionamento do CP no forno elétrico, os mesmos foram distribuídos aleatoriamente e suas posições registradas para posterior avaliação de tendências ou impactos no resultado final.

Foram ensaiados $15 \mathrm{CPs}$ de cada mistura, sendo 3 exemplares para cada combinação de variável independente (ambiente - sem aquecimento; $400^{\circ} \mathrm{C}$ - aquecimento rápido; $400^{\circ} \mathrm{C}$ - aquecimento lento, $800^{\circ} \mathrm{C}$ - aquecimento rápido; $800^{\circ} \mathrm{C}$ aquecimento lento). 


\subsubsection{Determinação da Resistência Mecânica}

A determinação da resistência mecânica residual foi realizada $24 \mathrm{~h}$ após o início do procedimento de aquecimento, de forma que a houvesse uma estabilização térmica dos CPs. Os mesmos foram rompidos em uma prensa hidráulica marca Shimadzu, com capacidade de $20000 \mathrm{kN}$. Os CPs foram posicionados de forma que as faces paralelas que estavam em contato com a forma fossem separadas do prato da prensa, somente, por dois pedaços de couro batido.

Destaca-se que a taxa de carregamento seguiu as recomendações da Norma EN12390-3 "Compressive strength of test specimens", pois não existe normatização para ensaio de CPs cúbicos no Brasil. Esta norma define uma taxa de carregamento entre a faixa de 0,1 a 2 MPa/s. Segundo a NBR 5739:2018 - "Concreto - Ensaio de Compressão de Corpos de Prova Cilíndricos", a taxa ser de 0,45 Mpa/s. Desta forma, foi utilizada a taxa de $0,45 \mathrm{Mpa} / \mathrm{s}$, para o ensaio dos CPs ensaiados neste trabalho.

\subsubsection{Análise Estatística}

Com o objetivo de avaliar se haviam diferenças significativas entre os grupos de variáveis analisadas foi realizada uma análise estatística, no software Statistic 10.0. Para tanto foi executada três análises ANOVA, de variância, uma para verificar a influência do efeito da taxa de aquecimento e da posição dos cps no forno, utilizando somente os resultados de resistência dos cps aquecidos, isto é, sem considerar os resultados dos cps expostos a temperatura ambiente. Para as outras duas análises foram utilizados os dados dos cps exposto a $23^{\circ} \mathrm{C}, 400^{\circ} \mathrm{C}$ e $800^{\circ} \mathrm{C}$ com taxa de aquecimento rápido e com taxa lenta. Destaca-se que a indicação para avaliação da resistência residual é utilizar aquecimento lento, para garantia que toda massa do cp esteja exposta à temperatura especificada, porém para a análise comparativa a análise foi executada para as duas taxas de aquecimento avaliada.

\section{RESULTADOS E DISCUSSÕES}

Os resultados do presente trabalho serão apresentados em três etapas distintas: comportamento quanto ao spalling, resistência mecânica residual dos corpos de prova aquecidos e avaliação de significância das variáveis independentes (tipo de mistura, temperatura de exposição, taxa de aquecimento e posição dos corpos de prova dentro do forno) através de análise estatística.

\subsection{Spalling}

Apesar dos CPs terem permanecido por, pelo menos, 2 anos em sala climatizada, com UR de $50 \% \pm 4 \%$ e temperatura de $23^{\circ} \mathrm{C} \pm 2^{\circ} \mathrm{C}$, os três corpos de prova da mistura de referência 0,30 sofreram spalling quando expostos a temperatura de $800^{\circ} \mathrm{C}$ e taxa de aquecimento rápida, conforme pode ser observado na Figura 5.

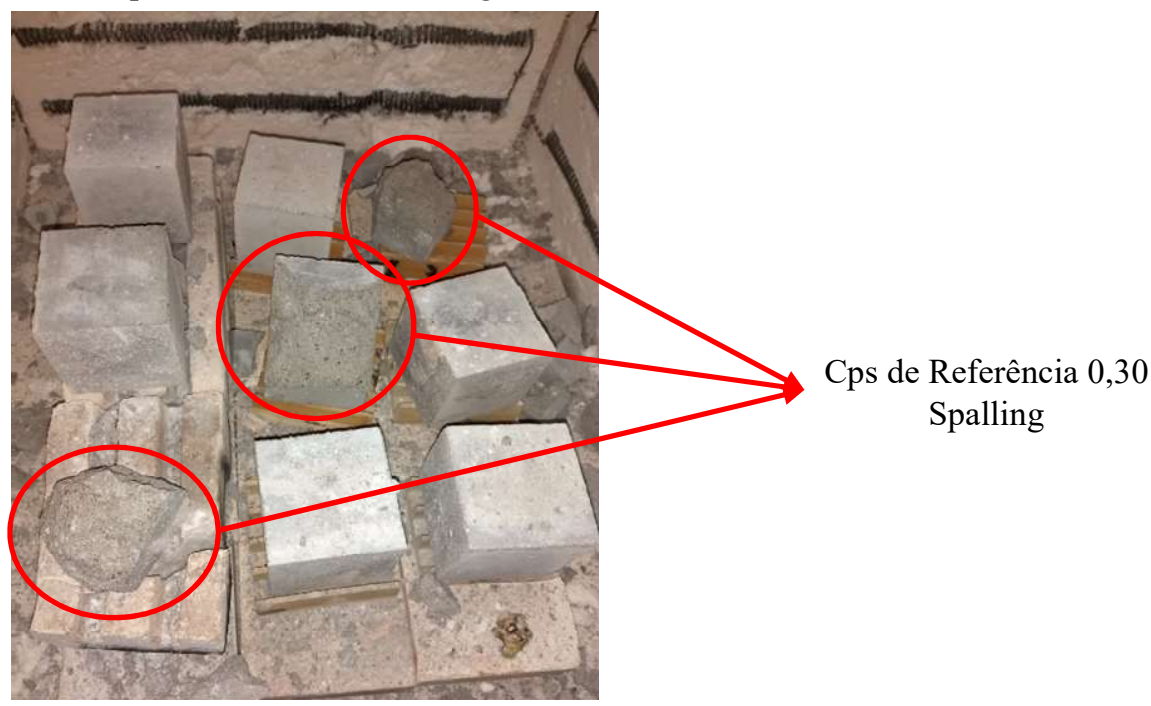

Figura 5: Vista do spalling nos CPs de referência 0,30.

Por acreditar que os corpos de prova estivessem em equilíbrio hidráulico, por terem permanecido em estabilização por 2 anos e por possuírem reduzidas dimensões, o que facilitaria a estabilização, a ocorrência do spalling não era esperada. $\mathrm{O}$ 
spalling ocorreu nos cps da mistura de referência de menor relação a/c, que possivelmente possui maior compacidade e por isto, maior dificuldade da saída de água para o meio ambiente. Outro fator importante é que a presença dos agregados porosos pode ter contribuído de forma positiva para o alívio de tensão gerada pela evaporação de água presente na mistura.

\subsection{Resistência Mecânica Residual}

Posterior aquecimento e ruptura dos corpos de prova os resultados foram avaliados e estão apresentados na Tabela 2 e Figura 6. Os dados apresentados é a média de dois ou três CPs prismáticos. Apesar de terem sido ensaiados 3 cps por combinação de variáveis independentes, após análise de espúrios, foi necessário excluir cinco resultados da análise, sendo 1 Ref $0,30\left(23^{\circ} \mathrm{C}\right)$, um $\operatorname{Ref} 0,30\left(400^{\circ} \mathrm{C}\right.$ - rápido $)$, um coríndon $\left(400^{\circ} \mathrm{C}\right.$ - rápido $)$, um ref $0,366\left(400^{\circ} \mathrm{C}-\right.$ lento $)$ e um cinza $\left(400^{\circ} \mathrm{C}\right.$ - Lento). Para análise dos espúrios foi seguida a metodologia abaixo.

1) Inicialmente eram determinadas a média, o desvio padrão e coeficiente de variação do grupo de dados relativo aos ensaios de resistência à compressão de cada material

2) Caso os valores de coeficiente de variação calculados fossem inferiores a $10 \%$, todos os dados do grupo eram levados em consideração;

3) Caso os valores de coeficiente de variação fossem superiores a10\%, o valor mais afastado da média do grupo era considerado como suspeito e era retirado da amostra para cálculo de uma nova média e de um novo desvio padrão. Caso a diferença absoluta entre o valor individual e essa nova média fosse superior a duas vezes o desvio padrão, o valor era definitivamente removido, sendo considerado espúrio. O processo era repetido até que não existissem mais valores possivelmente espúrios.

Tabela 2: Valores médios de resistência à compressão dos cubos.

\begin{tabular}{c|c|c|c|c|c}
\hline \multicolumn{7}{c}{ Resistência à Compressão [MPa] } \\
\hline & $23^{\circ} \mathrm{C}$ & $\begin{array}{c}400^{\circ} \mathrm{C} \\
\left(2^{\circ} \mathrm{C} / \mathrm{min}\right)\end{array}$ & $\begin{array}{c}800^{\circ} \mathrm{C} \\
\left(2^{\circ} \mathrm{C} / \mathrm{min}\right)\end{array}$ & $\begin{array}{c}400^{\circ} \mathrm{C} \\
\left(27,5^{\circ} \mathrm{C} / \mathrm{min}\right)\end{array}$ & $\begin{array}{c}800^{\circ} \mathrm{C} \\
\left(27,5^{\circ} \mathrm{C} / \mathrm{min}\right)\end{array}$ \\
\hline Ref 0,30 & 73,29 & 63,65 & 36,32 & 84,86 & 0,00 (spalling) \\
\hline Ref 0,36 & 73,56 & 48,06 & 26,80 & 68,63 & 35,46 \\
\hline Casca & 79,02 & 55,27 & 31,35 & 73,36 & 37,97 \\
\hline Cinza & 58,83 & 41,67 & 14,97 & 61,13 & 24,66 \\
\hline Corindon & 73,16 & 57,76 & 32,64 & 70,44 & 41,24 \\
\hline
\end{tabular}

Analisando os dados da Tabela acima, percebe-se que o valor médio da resistência à compressão da mistura de referência 0,30 à temperatura ambiente apresenta resultado muito abaixo do esperado. $\mathrm{O}$ valor foi praticamente o mesmo do que para mistura de referência 2 que possui relação a/c 0,066 superior, devendo esta possuir valor inferior. Além disto, a resistência média para a mistura ref 0,30 , quando exposta à $400^{\circ} \mathrm{C}$ e aquecimento rápido foi de $84,86 \mathrm{MPa}$, o esperado era que, para uma mesma mistura a resistência à compressão fosse maior para os cps não aquecidos. Por fim, destaca-se que aos 28 dias após a mistura, cps cilíndrico de $5 \mathrm{~cm}$ de diâmetro foram rompidos e a resistência média foi de $89,14 \mathrm{MPa}$, desta forma acredita-se que os cubos devem possuir resistência mínima de $90 \mathrm{MPa}$. Durante a ruptura percebeu-se problemas nas moldagens dos cps, o que provavelmente comprometeram o valor final.

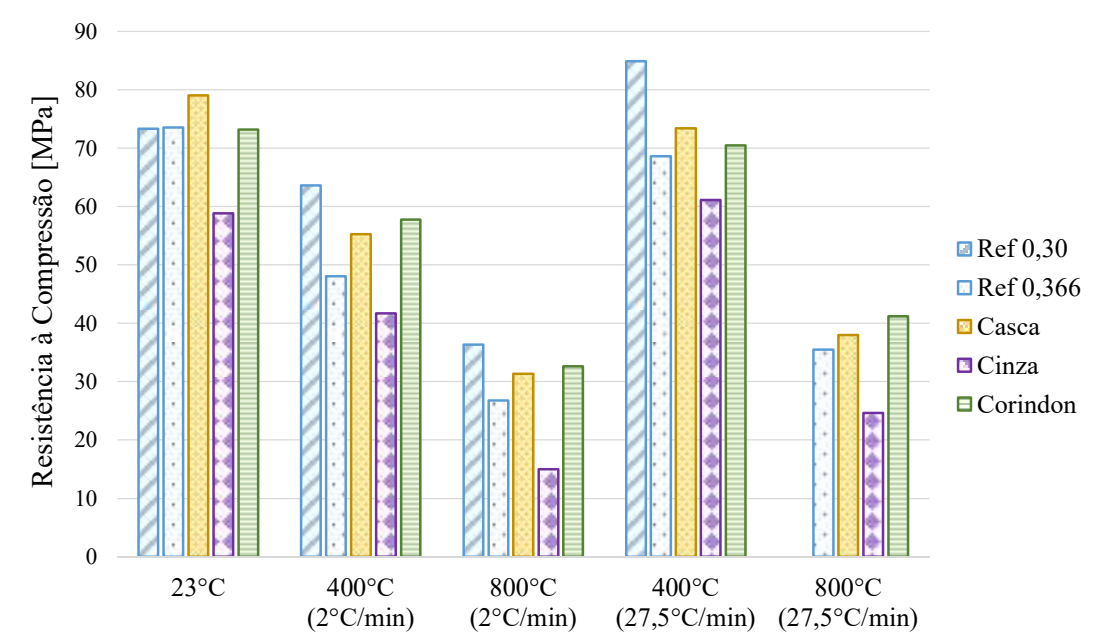

Figura 6: Resultados médios da resistência à compressão dos cps ensaiados. 
Analisando e comparando os resultados para as diferentes taxas de aquecimento verifica-se que taxas mais lentas reduzem as resistências de forma mais significativa. Enquanto, para o aquecimento de $400^{\circ} \mathrm{C}$ e aquecimento rápido o percentual máximo de redução da resistência foi de 7,2\% para a mistura com casca, para o mesmo patamar de temperatura, porém para taxa lenta a redução foi de até $34,7 \%$ para a mistura de referência 0,366 . Para a temperatura de $800^{\circ} \mathrm{C}$ percebe-se que a mistura que apresentou maior queda sob efeito do calor foi a com cinza, com queda de $58 \%$ para aquecimento rápido e de 74,5\% para aquecimento lento. Para este patamar de temperatura as reduções médias foram de $51,4 \%$ e de $63,5 \%$ para as taxas rápidas e lentas, respectivamente. Destaca-se que para as análises de redução não foram levados em consideração os valores dos cps de referência 0,30 , por considerar suspeito os sem aquecimento.

\subsection{Análise Estatística}

Após a primeira análise de variância verificou-se que o tipo de mistura e a posição de colocação dos cps no forno não apresentaram diferenças significativas entre grupos. Esta informação é pertinente, pois garante que o posicionamento dos cps não provocam alterações significativas na resistência à compressão, possivelmente por haver uma adequada distribuição de calor no interior do forno. As variáveis temperatura e taxa de aquecimento apresentaram diferenças significativas. Na Figura 7 é possível observar que a taxa de aquecimento de $2^{\circ} \mathrm{C} / \mathrm{min}$ tende, de forma geral, a fornecer valores de resistência 17,8\% menores do que os cps aquecidos com taxa de aquecimento conforme curva ISO, retirando da análise os cps de referência 1, que foram consideramos como tendo resistência 0 (zero) a diferença é ainda maior, resistência $26 \%$ maior para os cps aquecidos com taxa de $27,5^{\circ} \mathrm{C} / \mathrm{min}$. Salienta-se que esta diferença é menor para a temperatura de $800^{\circ} \mathrm{C}$, alcançando uma diferença de $17,8 \%$ entre as taxas de aquecimento investigadas.

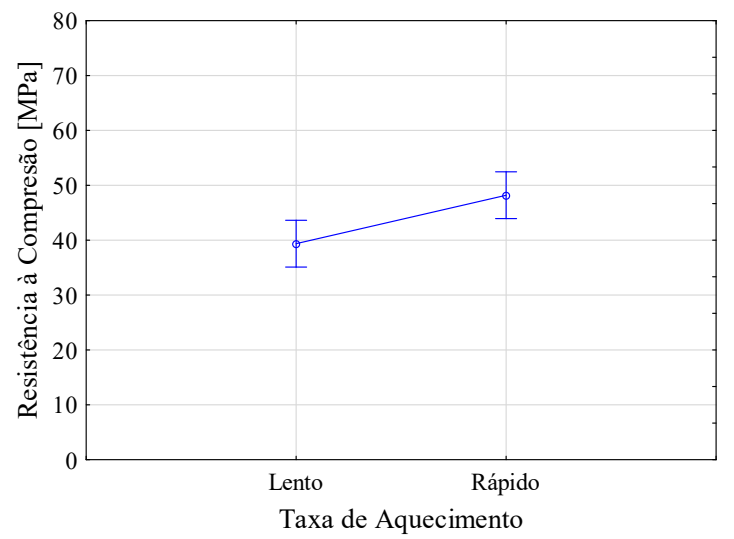

Figura 7: Resultados médios da resistência à compressão dos cps ensaiados.

Nas outras duas análises executadas para verificação do efeito da temperatura na resistência à compressão, observou-se que a maior diferença de comportamento foi para a temperatura de $400^{\circ} \mathrm{C}$. Na Figura 8 é possível verificar que para a taxa de aquecimento rápida, a média geral das resistências foi praticamente a mesma do que a médias dos corpos de prova sem aquecimento, enquanto que para a taxa de aquecimento lenta a redução foi de $25,6 \%$ em relação aos cps sem aquecimento.

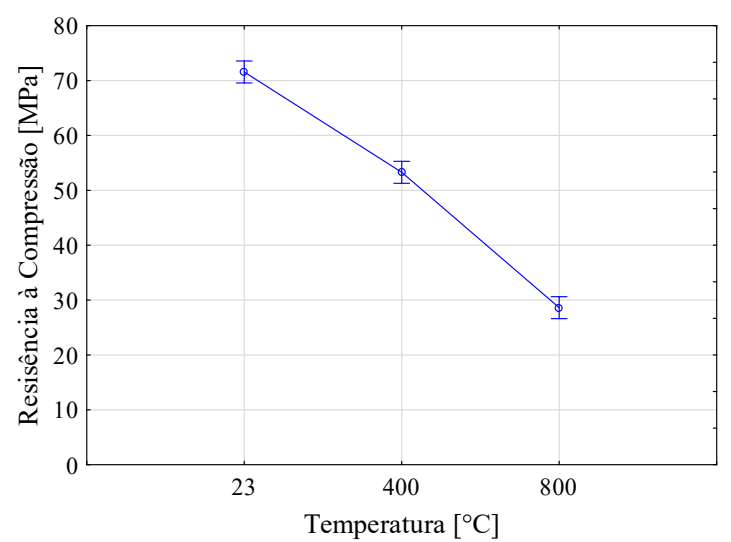

(a)

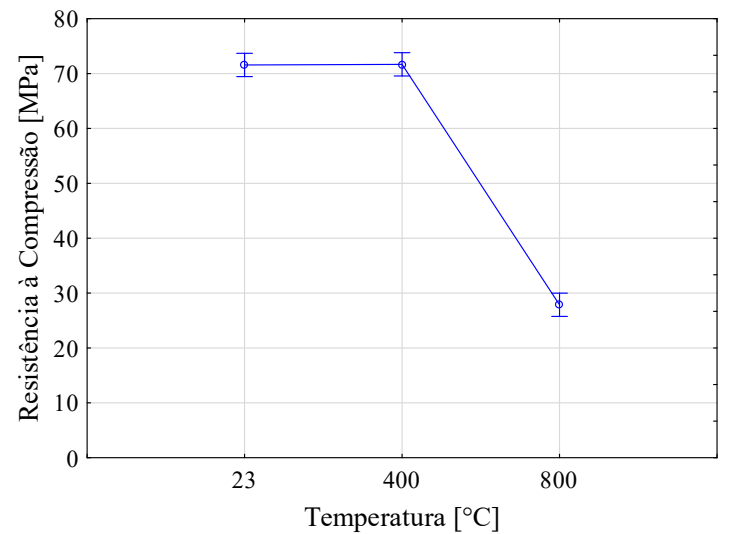

(b)

Figura 8: Perda de resistência à compressão versus temperatura, em função da taxa de aquecimento. (a) Lento; (b) rápido. 


\section{CONCLUSÕES}

Após análise dos dados apresentados neste trabalho verifica-se que a taxa de aquecimento influencia significativamente tanto o comportamento de spalling quanto a resistência residual de misturas cimentícias em altas temperaturas, por isto, é fundamental definir este parâmetro de ensaio em função dos objetivos do trabalho. Isto corroboram com Khoury et al. (2002) que indicam como um dos fatores que afetam a resistência residual do concreto em altas temperaturas sendo a taxa de aquecimento. A taxa de aquecimento e o tempo de patamar na temperatura especificada influenciam no alcance da temperatura ao longo do volume dos corpos de prova, isto é, se todo o volume é submetido à temperatura alvo. A grande diferença de comportamento entre as diferentes taxas de aquecimento, para a temperatura de $400^{\circ} \mathrm{C}$, ocorreu, provavelmente, pela temperatura alvo teve mais influência na parte superficial dos CPs, não atingindo o núcleo. Acreditase que para temperaturas mais altas, o dano é tão severo que a taxa de aquecimento, provoca alteração de comportamento, embora de menor magnitude.

Em relação à ocorrência de spalling, foi observado apenas na situação de aquecimento rápido, para o patamar de $800^{\circ} \mathrm{C}$ e na mistura de maior compacidade. A pesar da mistura de referência 0,30 ser mais compacta é possível que não tido mais dificuldade de perda da umidade ao longo dos 2 anos de estabilização. Com a elevação da temperatura a água presente tenta evaporar formando fissuras no interior do $\mathrm{CP}$, paralelas à superfície, ocorrendo o arranchamento explosivo das camadas superficiais.

\section{REFERÊNCIAS}

ALMEIDA, D. F. A. Patologia, Terapia e Profilaxia nas Construções. In: $19^{\circ}$ SIMPATCON - Simpósio de Aplicação da Tecnologia do Concreto, Campinas, São Paulo, 1998.

ALMEIDA, J. M. A. Comportamento em altas temperaturas e na reidratação de concretos convencional e com cinza de casca de arroz. 2017. 344 f. Tese (Doutorado em Engenharia Civil) Programa de Pós Graduação de engenharia civil, Universidade Federal do Rio Grande do sul, Porto Alegre, 2017.

ASSMANN, A. Physical properties of concrete modified with superabsorbent polymers. 2013. 213 f. Tese (Doutorado em Engenharia Civil e Ambiental) - Instituto de Materiais em Construção na Universidade de Stuttgart, Universidade de Stuttgart, Stuttgart, 2013.

AMERICAN SOCIETY FOR TESTING AND MATERIALS C 1761: Specification for lightweight aggregate for internal curing of concrete. West Conshohocken, 2015.

ASSOCIAÇÃO BRASILEIRA DE NORMAS TÉCNICAS. NBR 13276: Argamassa para assentamento e revestimento de paredes e tetos - Determinação do índice de consistência. Rio de Janeiro, 2016.

2009.

.ABNT NM 52: Agregado miúdo - Determinação da massa específica e massa específica aparente. Rio de Janeiro,

NBR 5739: Concreto Ensaio de compressão de corpos de prova cilíndricos. Rio de Janeiro, 2018.

BENTUR, A. Introduction: Overview of early age cracking. In: BENTUR, A. Early Age Cracking in Cementitious Systems. RILEM, 2000.

CAetano, L. F. Avaliação do Potencial de uso de Materiais Porosos como Agentes para Cura Interna de Concretos com Reduzida Relação a/c. 2019. 270 f. Tese (Doutorado em Engenharia Civil) Programa de Pós Graduação de engenharia civil, Universidade Federal do Rio Grande do sul, Porto Alegre, 2019.

COSTA, C. N.; FIGUEIREDO, A. D.; SILVA, V. P. Aspectos tecnológicos dos materiais de concreto em altas temperaturas. NUTAU 2002 - Sustentabilidade, Arquitetura, Desenho Urbano. USP, S. Paulo, 2002.

EUROPEAN COMMITTEE FOR STANDARDIZATION EN 123903. Testing hardened concrete. Compressive strength of test specimens. 2019. 
GALleTO, A.; MENEGUINI, E. C. A. Comportamento do concreto submetido à temperaturas elevadas. Seminário apresentado à disciplina Análise Experimental de Estruturas - FEC-UNICAMP, Campinas, 2000.

HAGER, I. Behaviour of cement concrete at high temperature. Bulletin of the Polish Academy of Sciences. Technical Sciences, v. 61, n. 1, 2013.

HERTZ, K.D. Danish investigations on silica fume concretes at elevated temperatures. ACI Materials Journal, Denmark, v. 89, n. 4, p. 345-347, 1992.

INTERNATIONAL STANDARDIZATION FOR ORGANIZATION. ISO 834:2014: Fire resistance tests. Geneva, 1999.

KIRCHHOF, L. D. Estudo teórico-experimental da influência do teor de umidade no fenômeno de spalling explosivo em concretos expostos a elevadas temperaturas. Tese (Doutorado em Engenharia Civil), Universidade Federal do Rio Grande do Sul, Porto Alegre, RS, 2010.

KOVLER, K; JENSEN, O. M. Introdution. In: RILEM, State-of-the-art report of the Rilem Technical Committee 196-ICC: Internal curing of concrete. Ed. Springer, Cap. 1, 2007.

LEE, H. K.; JANG, J. G.; AHN, Y. B.; Mechanical properties of lightweight concrete made with coal ashes after exposure to elevated temperatures. Cement and Concrete Research, v. 72, p. 27 32, 2016.

LURA, P. Autogenous deformation and internal curing of concrete. 2003. 208 f. Tese (Doutorado em Engenharia Civil) - Programa de Pós-Graduação, Delft University of Technology, Delft, 2003.

MANZANO, M. A. R. Estudo experimental de materiais cimentícios de alta resistência modificados com polímeros superabsorventes (psas) como agentes de cura interna. 2016. 351 f. Tese (Doutorado em Estruturas e Construção Civil) - Departamento de engenharia civil e ambiental, Faculdade de Tecnologia, Universidade de Brasília, Brasília, 2016.

PENG, G. F. et al. Explosive spalling and residual mechanical properties of fiber toughened high performance concrete subjected to high temperature s. Cement and Concrete Research , v. 36, p. 723 727, 2006.

POWERS, T. C. Capillary continuity or discontinuity in cement pastes. Journal of the PCA Research and Development Laboratories, v. 1, n. 2, p. 38-48, 1959.

POWERS, T. C. Structure and Physical Properties of Hardened Portland Cement Paste Authorized Reprint from American Ceramic Society. Journal of The American Ceramic Society, [s. 1.], v. 41, n. 1958, p. 1-7, 1958.

POWERS, T.C; BROWNYARD, T.L. Studies of the Physical properties of Hardened Cement Paste. Journal of the American Concrete Institute, v. 43, 1947

RILEM Technical Committe. RILEM TC 129-MHT: Test methods for mechanical properties of concrete at high temperatures. 2000 .

RILEM Technical Committe. RILEM TC 225-SAP: Application of super absorbent polymers in concrete construction. 2014.

SANTOS, C. C. Propriedades mecânicas residuais após incêndio de betões normais. Tese (Doutorado em Engenharia Civil), Universidade de Coimbra, Coimbra, 2012.

SENSALE, G. R.; GONÇALVES, A. F. Effects of Fine LWA and SAP as Internal Water Curing Agents. International Journal of Concrete Structures and Materials, v.8, n.3, p. 229-238, 2014.

PAULON, V. A. Durabilidade do Concreto - Avanços da Tecnologia. In: $7^{\circ}$ SIMPATCON - Simpósio de Aplicação da Tecnologia do Concreto, Campinas, São Paulo, 1984. 